\title{
Physics-Based Subsurface Visualization of Human Tissue
}

\author{
Richard Sharp ${ }^{1}$, Jacob Adams ${ }^{1}$, Raghu Machiraju ${ }^{1}$, Robert Lee ${ }^{1}$, and Robert Crane ${ }^{2}$
}

\begin{abstract}
In this paper, we present a framework for simulating light transport in three dimensional tissue with inhomogeneous scattering properties. Our approach employs a computational model to simulate light scattering in tissue through the finite element solution of the diffusion equation. Although our model handles both visible and non-visible wavelengths, we especially focus on the interaction of near infra-red (NIR) light with tissue. Since most human tissue is permeable to NIR light, tools to non-invasively image tumors, blood vasculature and monitor blood oxygenation levels are being constructed. We apply this model to a numerical phantom to reproduce visually the images generated by these real world tools. Therefore, in addition to enabling inverse design of detector instruments, our computational tools produce physically-accurate visualizations of subsurface structures.
\end{abstract}

Index Terms - volume rendering, near infrared, biological tissue, light scattering

\section{INTRODUCTION}

Much of the literature in optical models and mechanisms for light transport has been devoted to the visible part of the electromagnetic spectrum. These models have formed the basis of many volume rendering algorithms including raycasting, splatting, and so on. Recently a variety of wavelengths, including near-infrared (NIR), have been increasingly used in non-invasive imaging. Unlike with visible wavelengths of light, human tissue is extremely permeable to NIR light [10], such as that generated by a TV remote control. Researchers have leveraged this property to develop methods for noninvasive imaging and detection to locate tumors, determine blood oxygenation levels, assess nutritional absorption, brain activity, water content, and many others [5], [16], [17], [19], [24], [35], [44]. Unfortunately, current models of volume rendering do not accurately capture the diffuse nature of NIR transport in tissue.

In this paper we introduce an optical and biological model for volume visualization which mimics the optical scattering seen by the NIR transillumination device (Figure 1) developed by InfraRed Imaging Systems, Inc. This device, called the IRIS Vascular Viewer, is used by medical personnel to detect the subsurface location of arteries to draw arterial blood taps. A major concern is the quality of the image, which can be significantly different depending on the patient. There may be anatomical differences as well as differences in the optical scattering properties of tissues, which may vary depending on the patient's age, sex, and weight. Numerical simulations, along with accurate numerical phantoms ${ }^{1}$, offer

\footnotetext{
${ }^{1}$ The Ohio State University, ${ }^{2}$ InfraRed Imaging Systems, Inc.

${ }^{1}$ The term "numerical phantom" refers to the geometry and scattering properties of the volume to which the numerical simulation will be applied.
}

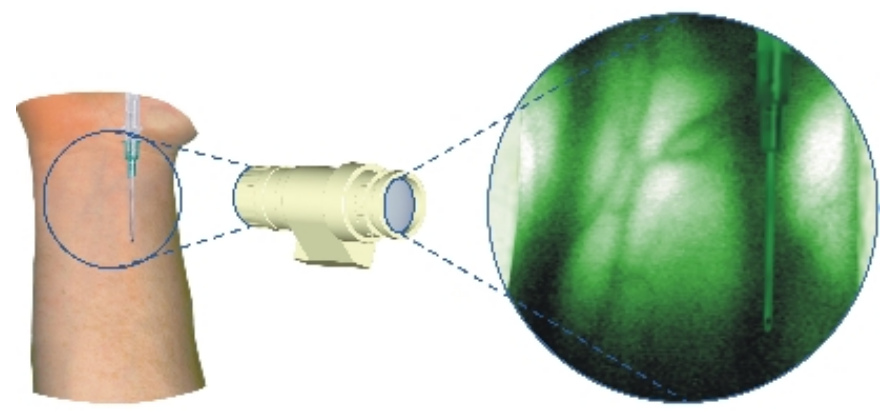

Fig. 1. This figure illustrates the use of the Vascular Viewer ${ }^{\mathrm{TM}}$ which illuminates the far side of the forearm with an array of infrared LEDs, shines the light through the arm, then is viewed directly using a filtered infrared scope.

possibly the only way to study the NIR propagation through tissue since it provides volumetric light intensity data which cannot be obtained from measurements. Simulations to study the light interaction with the tissue in the volume may be critical in designing not only NIR devices but also postprocessing algorithms to improve the imaging. Secondly, once an optical model exists, simulations can be done with any set of parameters. Doing this in a real world experiment would be very difficult since one must capture the anatomy and characterize all the NIR tissue properties for a large number human subjects. A similar type of study was done with Laney et al. [27] in the simulation of radiography for high-energy physics experiments. Like our model, the intent of these authors is to provide a numerical basis for designing experiments, validating other simulations, and understanding the results of real world experiments.

There is a need for viable optical models and computational methods that study light transport through biological tissue at a variety of scales and wavelengths. Most existing optical models [32] and volume rendering methods based on those do not display internal structure in a realistic manner since they do not simulate the high amount of scattering that occurs. However, in computer graphics literature, subsurface scattering methods have been employed to increase the realism of organic surfaces and human skin [23]. Unfortunately, these methods are not suited to the kinds of diffusion seen with NIR light because they rely on the fact that at visible wavelengths the effects of subsurface scattering are relatively local (when a visible wavelength photon enters the surface of human skin, it is absorbed after a few millimeters if it does not exit before then). When NIR light scatters through human tissue the effects are global (a photon which enters one side of a 
(a)

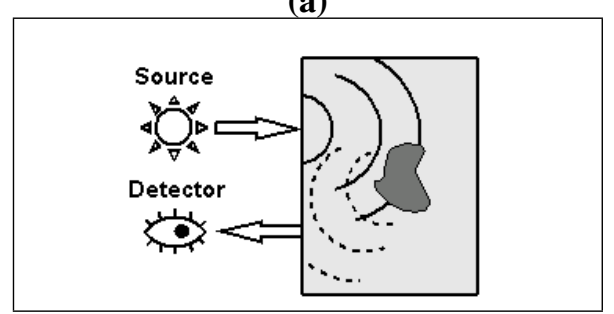

(b)

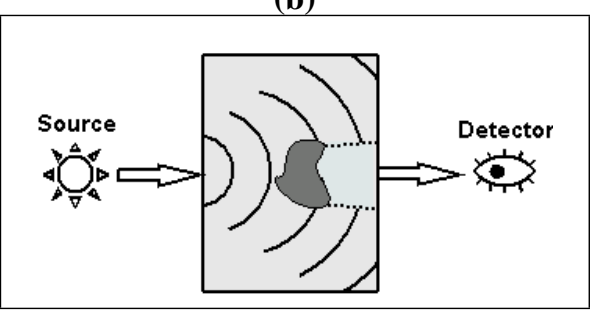

Fig. 2. (a) source detector setup to measure backscattered light; (b) source detector setup for transillumination.

human arm can easily travel several centimeters before being completely absorbed). These models would become computationally cumbersome to simulate such extensive scattering. thus they can not feasibly be used for the purposes described below.

Over the past decade, there have been great advancements in simulating light transport using the finite element method (FEM), but there have been very few attempts at a full 3D scattering to model the propagation of scattered light. In this work, we implement a full 3D FEM solution of the light diffusion equation. As a result, we can model the rich heterogeneity of tissue types and geometry that exists under the skin. Additionally, we suggest some optimizations that efficiently render of the underlying model.

\section{A. NIR Transillumination Devices}

Many NIR measurement applications measure the amount of backscattering from a source with a detector located on the same side (see Figure 2a). While this method can measure many useful properties of tissues it is limited to only a depth of a few millimeters with NIR wavelengths. For many applications (such as measuring blood oxygenation levels) a limited depth is not a restriction, however to visualize structures deep within tissue requires a transillumination configuration (Figure 2b). In this scenario the source is placed on the far side of the tissue and the NIR light is scattered completely through the extremity to the far side. Objects which absorb NIR light and which are close to the surface on the far side are then seen as shadows by the detector. The detector used to generate the real world images (Figures $3 b$ and 1) is a NIR viewing scope.

There are many uses for a transillumination source detector configuration. Traditionally physicians have used transillumination to shine visible light through cavity or organs of premature or newborn infants. However, as opposed to with visible light, NIR light one can see deeper structures such as veins or arteries. This real-time noninvasive visualization is useful for clinicians to identify a patient's vein or artery for vasculature taps. Without visualization, clinicians rely on what they can physically observe and feel through the skin which is not always sufficient: $28 \%$ of intravenous (IV) taps in normal adults fail on the first attempt [7], and $43 \%$ of IVs require three or more insertion attempts in pediatrics [29]. These IV insertion failures not only cause discomfort for the patient, but the alternatives drastically increase the cost of treatment and the failures can lead to complications including infection. With real time visualization the risk of missing a vein or artery due to lack of knowledge of its position decreases, since the clinician can see the subsurface location of the vessel.

In our work, we specifically focus on simulating the transillumination source detector configuration shown in Figure $2 b$. An accurate physics-based visualization is the only recourse to obtain a rendering which displays the internal structures in their true form. Consider the image in Figure 3a; this image was created through our 3D simulation with a numerical phantom resembling a human forearm. The shadowing effects caused by the subsurface vasculature are well depicted. A typical volume rendering or a method that strictly uses only forward scattering [26] will not result in a similar image since the light will not be diffused properly in the medium.

(a)

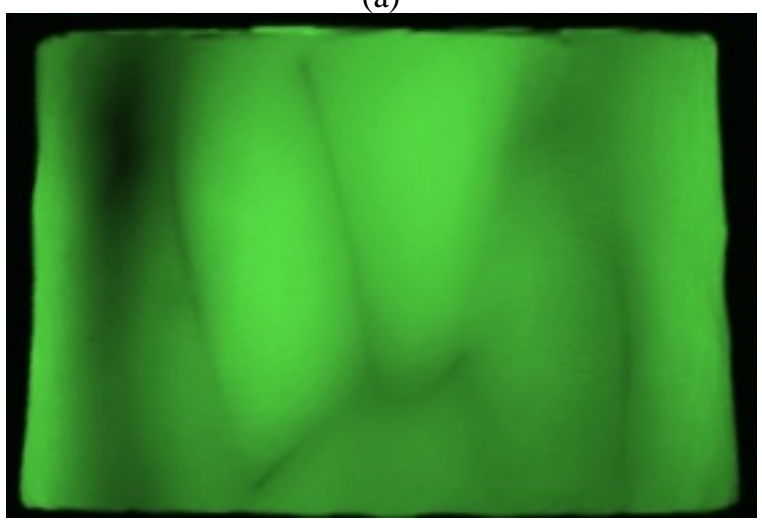

(b)

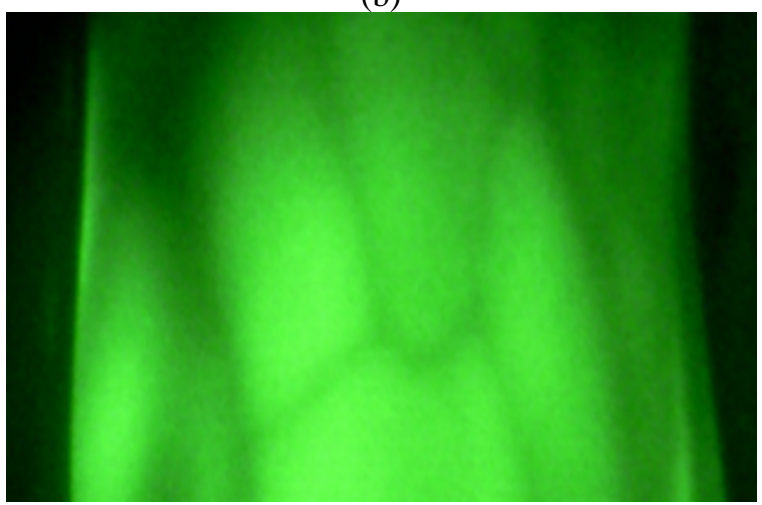

Fig. 3. Figure (a) shows our simulation of near infrared light through an arm model where the geometry, major internal veins, and arteries were segmented from MRI data. Superficial veins on the surface were added manually from the known locations on the original specimen. (Figure 5 shows the original MRI dataset while Figure 9 shows a perspective view of this dataset.) Figure (b) shows the result from the Vascular Viewer ${ }^{T M}$ for the same arm.

Our model simulates the NIR light diffusion effects by solving a light diffusion equation with the finite element method (FEM) on a 3D tetrahedral mesh. Thus the simulation 
is dependent not only on the mathematical model, but also on an accurate numerical phantom. In this paper we present a phantom which we have created based on an MRI of the primary author's arm, as well as the visible surface veins (see Figure 3). This model allowed us to verify the visual effect created by both deep subsurface structures and structures near the surface. We have also included a simple procedural mesh which is used to conduct experiments on possible artery depths (see Figure 10).

In the next section we will detail previous work in the area of light propagation modeling. In Section III we sketch the theoretical basis for our optical model for light scattering in tissue while in Section IV we derive the finite element solution method we use for solving the system. Next, in Section V we briefly discuss our numerical phantom and in Section VI we present our implementation of this formulation to include an efficient precalculation of the scattering for fast light position updates. Finally, in Section VII we present our results with this model and discuss our conclusions in Section VIII.

\section{Previous Work}

Recently many practical computational subsurface scattering models have been introduced in a drive to increase the quality of realistic image synthesis in computer graphics [8], [22], [23], [26], [34], [38]. Although we do not attempt to simulate scattering of light for visible wavelengths here, many of the principles are similar across visible and non-visible wavelengths.

The work developed by Jensen et al. [23] has arguably driven subsurface scattering research in recent years. The approximation presented by the authors uses a dipole solution to the diffusion equation to efficiently calculate local subsurface scattering. This model was later enhanced to efficiently calculate the effects of diffusion using a spatial partitioning technique [22]. Although more work was published that handles multilayer materials [12] the dipole model still cannot handle non-local scattering effects or non-layered discontinuities like those presented in this paper.

A similar work developed by Riley et al. [34] uses an approximation to a forward scattering model to simulate light scattering in clouds. In a trade off for runtime performance this model only propagates light in a forward direction and is limited to narrowly peaked forward biased phase functions. Although most human tissue does forward-scatter light, there are some tissues whose mean cosine of the phase function is less forward-scattering than that of clouds [10]. Furthermore, at the interfaces of subsurface discontinuities there can be large amounts of reflection which would be completely lost in a model which only propagates light in a forward direction.

There has been an increasing interest in the biomedical imaging field to develop techniques for NIR spectroscopy [2], [44]. Wilson et. al present a method for optical spectroscopy in determining the depth of sub-tissue level features in [43]. To reduce the noise in backscattered optical images the authors gate photons which have only a single scattering event and perform experiments using modulated light. Arridge and Hebden propose a method for optical tomography in [1].
Unfortunately optical tomography faces difficulty in obtaining a high signal to noise ratio when scattering light through tissue. Schweiger et al. introduces the finite element method for time domain light diffusion in [36] which discusses a variety of boundary conditions and two specialized source representations in two dimensions. Although the focus of our paper is on completely diffusive media, recent work in finite element light diffusion has focused on embedded non-diffusive regions [3], [4], [28]. Finally recent work in bioluminescence tomography has motivated a drive to understand the nature of scattering light in tissue [11].

Max [32] derives an optical model (among many) for volume rendering based on the transport equation derived by Chandrasekhar [9]. One can also consider the work in this paper to be diffusive volume rendering optical model. Max's solution method is to use an efficient implementation of the discrete ordinates method. This is necessary if scattering is not the main method of propagation in the medium. However, for human tissue light scattering is the dominant method of propagation. This is due to the fact that the rate at which tissue absorbs light is much less than the rate at which it scatters it. Thus the transport equation need not be solved directly. Instead, we approximate the transport equation by the diffusion equation and render the result from there.

\section{DifFusion THEORY OF Light TRANSPORT}

In general, the propagation of light in a medium at a point $\mathbf{r}$ and direction $\hat{\mathbf{s}}$ can be approximated by the Transport Equation. Here it is presented in the same form as Ishimaru [20]

$\frac{d I_{d}(\mathbf{r}, \hat{\mathbf{s}})}{d s}=-\mu_{t} I_{d}(\mathbf{r}, \hat{\mathbf{s}})+\frac{\mu_{t}}{4 \pi} \int_{4 \pi} p\left(\hat{\mathbf{s}}, \hat{\mathbf{s}}^{\prime}\right) I_{d}\left(\mathbf{r}, \hat{\mathbf{s}}^{\prime}\right) d \omega^{\prime}+\varepsilon_{r i}(\mathbf{r}, \hat{\mathbf{s}})$

where $I_{d}(\mathbf{r}, \hat{\mathbf{s}})$ is the diffuse intensity, $\mu_{t}$ is the extinction cross section, $p\left(\hat{\mathbf{s}}, \hat{\mathbf{s}}^{\prime}\right)$ is the phase function, $\varepsilon_{r i}(\mathbf{r}, \hat{\mathbf{s}})$ is the source function due to reduced incident intensity, $I_{r i}(\mathbf{r}, \hat{\mathbf{s}})$, which has the behavior

$$
\frac{d I_{r i}(\mathbf{r}, \hat{\mathbf{s}})}{d s}=-\mu_{t} I_{r i}(\mathbf{r}, \hat{\mathbf{s}})
$$

In the case of human tissue or any other highly scattering media the diffusion approximation gives accurate solutions which are computationally simpler to solve. The diffusion approximation is based on the assumption that light is completely scattered a small distance below the surface. In this case the intensity is not determined by direction, but is a scalar value defined at a point inside the medium. Formally, this quantity is the average diffuse intensity:

$$
U_{d}(\mathbf{r})=\frac{1}{4 \pi} \int_{4 \pi} I_{d}(\mathbf{r}, \hat{\mathbf{s}}) d \omega .
$$

To derive the diffusion approximation we first expand $I_{d}(\mathbf{r}, \hat{\mathbf{s}})$ in a Taylor series (of which the first term is $U_{d}$ ) and substitute only the first and second terms back into Equation 1. After several simplifications we are left with the diffusion approximation

$$
\nabla \cdot \beta \nabla U_{d}(\mathbf{r})-\lambda U_{d}(\mathbf{r})+S(\mathbf{r})=0
$$




\begin{tabular}{|c|l|}
\hline$p\left(\hat{\mathbf{s}}, \hat{\mathbf{s}}^{\prime}\right)$ & Phase function \\
$g$ & Mean cosine of the scattering angle \\
$\mu_{a}$ & Absorption cross section \\
$\mu_{s}$ & Scattering cross section \\
$\mu_{s}^{\prime}$ & Reduced scattering coefficient $\left(\mu_{s}(1-g)\right)$ \\
$\mu_{t}$ & Extinction cross section $\left(\mu_{a}+\mu_{s}\right)$ \\
$\mu_{t r}$ & Transport cross section $\left(\mu_{s}(1-g)+\mu_{a}\right)$ \\
$I_{d}(\mathbf{r}, \hat{\mathbf{s}})$ & Diffuse intensity \\
$I_{r i}(\mathbf{r}, \hat{\mathbf{s}})$ & Reduced incident intensity (Eq. 2) \\
$\varepsilon_{r i}(\mathbf{r}, \hat{\mathbf{s}})$ & Reduced incident intensity source \\
$U_{d}(\mathbf{r})$ & Average diffuse intensity (Eq. 3) \\
$U_{r i}(\mathbf{r})$ & Average reduced intensity \\
$\beta$ & Constant term $\left(\mu_{t r}\right)-1$ \\
$\lambda$ & Constant term $3 \mu_{a}$ \\
$\nu$ & Constant term $3 \mu_{s}$ \\
$V^{e}$ & volume of element $e$ \\
$\Delta_{f}$ & area of the face $f$ of element $e$ \\
$\mathbf{Q}_{\mathbf{1}}(\mathbf{r})$ & First order source distribution (Eq. 7) \\
\hline
\end{tabular}

TABLE I

TABLE OF VARIABLES

where $\beta$ and $\lambda$ are constant terms (see Table I) and

$$
S(\mathbf{r})=\nu U_{r i}(\mathbf{r})-\frac{3}{4 \pi} \nabla \cdot \beta \mathbf{Q}_{1}(\mathbf{r})
$$

where $\nu$ is a constant term (see Table I), $\mu_{t r}$ is the transport cross section, and

$$
U_{r i}(\mathbf{r})=\frac{1}{4 \pi} \int_{4 \pi} I_{r i}(\mathbf{r}, \hat{\mathbf{s}}) d \omega
$$

is defined as the average reduced incident intensity. The term $\mathbf{Q}_{1}(\mathbf{r})$ is the first order source distribution which is non-zero only when the phase function is anisotropic. Formally it is defined as:

$$
\mathbf{Q}_{\mathbf{1}}(\mathbf{r})=\frac{\mu_{t}}{\mu_{t r}} \int_{4 \pi}\left[\frac{1}{4 \pi} \int_{4 \pi} p\left(\hat{\mathbf{s}}, \hat{\mathbf{s}}^{\prime}\right) \hat{\mathbf{s}} d \omega\right] I_{r i}\left(\mathbf{r}, \hat{\mathbf{s}}^{\prime}\right) d \omega^{\prime} .
$$

Complete details of this derivation can be found in Ishimaru [20, pages $175-178]$.

\section{A. Boundary Conditions}

The exact boundary condition for the Transport Equation (Eq. 1) is that no diffuse intensity should enter the medium from the outside. Unfortunately, this boundary cannot be enforced on the diffusion equation since the intensity quantity, $U_{d}(\mathbf{r})$, is defined independently of direction. A common approximate boundary condition used by Ishimaru [20] can be derived by forcing the total inward directed flux to zero $\int_{2 \pi^{-}} I_{d}(\mathbf{r}, \hat{\mathbf{s}})\left(\hat{\mathbf{s}} \cdot \hat{\mathbf{n}}^{-}\right) d \omega=0$, where $\hat{\mathbf{n}}^{-}$is the inward directed normal at point $\hat{\mathbf{s}}$. This boundary condition can be formulated in terms of $U_{d}(\mathbf{r})$ alone as

$$
\mu_{t r} U_{d}(\mathbf{r})-\frac{2}{3} \frac{\partial}{\partial n^{-}} U_{d}(\mathbf{r})+\frac{2 \hat{\mathbf{n}}^{-} \cdot \mathbf{Q}_{1}(\mathbf{r})}{4 \pi}=0
$$

where $\partial / \partial n^{+}$is the normal derivative in the outward direction (see [20, pages 179-180] for the derivation).

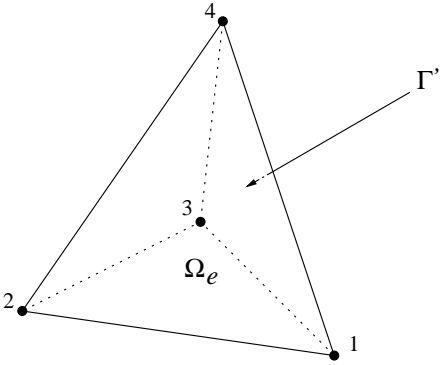

Fig. 4. An example three dimensional element $\Omega_{e}$ with nodes $1 \ldots 4$. The surface of this element is indicated by $\Gamma^{\prime}$ as described in Equation 9 .

\section{Finite Element Derivation}

This section describes the formulation of our FEM system of equations for the diffusion equation based on the method of weighted residuals [13]. It is necessary for us to pose our problem as light scattering through a mesh, rather than a local problem as Jensen et al. [23] do, since the nature of the scattering is global and the discontinuities in the material are non-layered.

The purpose of this section is to present only the steps useful for the implementation of our model. A description of our implementation follows in Section VI. Readers unfamiliar with the FEM can refer to the classic texts [45] and [39] for an in depth discussion of FEM theory. Algorithms VI-A and VI-B in Section VI refer the reader to the specific equations in this section necessary for implementation.

The discussion that follows assumes $\Omega_{e}$ is a tetrahedral element, $e$, shown in Figure 4. In the FEM we solve for the unknowns at the nodes and interpolate the solution as a summation of the node unknowns weighted by functions. For example if $U_{d}(\mathbf{r})$ is known at all four points we can solve for $U_{d}(\mathbf{r})$ at any point inside the element by $U_{d}(\mathbf{r})=$ $\sum_{j=1}^{4} U_{d(j)}^{e} \phi_{j}^{e}(\mathbf{r})$. The standard weighted residual expression based on Equation 4 for a single element $e$ is then given by

$$
\begin{aligned}
& -\int_{\Omega} \nabla \phi_{i}^{e}(\mathbf{r}) \cdot \beta \nabla U_{d}^{e}(\mathbf{r}) d \Omega-\int_{\Omega} \phi_{i}^{e}(\mathbf{r}) \lambda U_{d}^{e}(\mathbf{r}) d \Omega \\
& \quad+\int_{\Omega} \phi_{i}^{e}(\mathbf{r}) S^{e}(\mathbf{r}) d \Omega+\int_{\Gamma_{s}^{\prime}} \phi_{i}^{e}(\mathbf{r}) \beta\left(\frac{\partial}{\partial n^{+}} U_{d}^{e}(\mathbf{r})\right) d \Gamma^{\prime}=0
\end{aligned}
$$

for local nodes $i=1,2,3,4$, where $\Gamma_{s}^{\prime}$ refers to the boundary of element $e$. The last term in Equation 9, $\partial U_{d}^{e}(\mathbf{r}) / \partial n^{+}$ accounts for flux which is passed from one element to the other. For inter-element boundaries this term will cancel out since the flux is continuous. However, at the outer boundary the boundary condition given by Equation 8 must be properly enforced. Next, we expand $U_{d}(\mathbf{r})$ and $S$ in element $e$ in terms of the finite element basis functions, where $U_{d}$ is defined above and $S=\sum_{j=1}^{4} S_{j}^{e} \phi_{j}^{e}(\mathbf{r})$. Thus, ignoring the boundary term $\Gamma_{e}$ for now, we write Equation 9 in matrix form

$$
\left[K^{e}\right]\left\{U_{d}^{e}\right\}=\left\{w^{e}\right\}
$$

where $U_{d}^{e}=\left[U_{1 d}^{e}, U_{2 d}^{e}, U_{3 d}^{e}, U_{4 d}^{e}\right]^{T}$. The elements of $\left[K^{e}\right]$ can be calculated analytically as 


$$
\begin{aligned}
K_{i j}^{e} & =\int_{\Omega}\left[\beta \nabla \phi_{i}^{e}(\mathbf{r}) \cdot \nabla \phi_{j}^{e}(\mathbf{r})+\lambda \phi_{i}^{e}(\mathbf{r}) \phi_{j}^{e}(\mathbf{r})\right] d \Omega \\
& =\frac{\beta}{36 V^{e}}\left(b_{i}^{e} b_{j}^{e}+c_{i}^{e} c_{j}^{e}+d_{i}^{e} d_{j}^{e}\right)+\frac{V^{e}}{20} \lambda\left(1+\delta_{i j}\right)
\end{aligned}
$$

where $\delta_{i j}$ is the Kronecker delta ( 1 when $i=j$ and 0 otherwise) and $b_{i}^{e}, c_{i}^{e}$, and $d_{i}^{e}$ are constants derived from an analytical integration of the linear interpolation functions in Equation 11. Since the calculation of these constants in standard textbooks is often left to the reader we present the solution below

$$
\begin{aligned}
& b_{1}^{e}=y_{2}\left(z_{4}-z_{3}\right)+y_{3}\left(z_{2}-z_{4}\right)+y_{4}\left(z_{3}-z_{2}\right) \\
& b_{2}^{e}=y_{1}\left(z_{3}-z_{4}\right)+y_{3}\left(z_{4}-z_{1}\right)+y_{4}\left(z_{1}-z_{3}\right) \\
& b_{3}^{e}=y_{1}\left(z_{4}-z_{2}\right)+y_{2}\left(z_{1}-z_{4}\right)+y_{4}\left(z_{2}-z_{1}\right) \\
& b_{4}^{e}=y_{1}\left(z_{2}-z_{3}\right)+y_{2}\left(z_{3}-z_{1}\right)+y_{3}\left(z_{1}-z_{2}\right) \\
& c_{1}^{e}=x_{2}\left(z_{3}-z_{4}\right)+x_{3}\left(z_{4}-z_{2}\right)+x_{4}\left(z_{2}-z_{3}\right) \\
& c_{2}^{e}=x_{1}\left(z_{4}-z_{3}\right)+x_{3}\left(z_{1}-z_{4}\right)+x_{4}\left(z_{3}-z_{1}\right) \\
& c_{3}^{e}=x_{1}\left(z_{2}-z_{4}\right)+x_{2}\left(z_{4}-z_{1}\right)+x_{4}\left(z_{1}-z_{2}\right) \\
& c_{4}^{e}=x_{1}\left(z_{3}-z_{2}\right)+x_{2}\left(z_{1}-z_{3}\right)+x_{3}\left(z_{2}-z_{1}\right) \\
& d_{1}^{e}=x_{2}\left(y_{4}-y_{3}\right)+x_{3}\left(y_{2}-y_{4}\right)+x_{4}\left(y_{3}-y_{2}\right) \\
& d_{2}^{e}=x_{1}\left(y_{3}-y_{4}\right)+x_{3}\left(y_{4}-y_{1}\right)+x_{4}\left(y_{1}-y_{3}\right) \\
& d_{3}^{e}=x_{1}\left(y_{4}-y_{2}\right)+x_{2}\left(y_{1}-y_{4}\right)+x_{4}\left(y_{2}-y_{1}\right) \\
& d_{4}^{e}=x_{1}\left(y_{2}-y_{3}\right)+x_{2}\left(y_{3}-y_{1}\right)+x_{3}\left(y_{1}-y_{2}\right)
\end{aligned}
$$

where $x_{n}, y_{n}$, and $z_{n}$ are the $x, y$, and $z$ coordinates of node $n$. Finally, $V^{e}$ is the volume of element $e$

$V^{e}=\frac{1}{6}\left|\begin{array}{l}x_{1}\left[y_{2}\left(z_{4}-z_{3}\right)+y_{3}\left(z_{2}-z_{4}\right)+y_{4}\left(z_{3}-z_{2}\right)\right]+ \\ x_{2}\left[y_{1}\left(z_{3}-z_{4}\right)+y_{3}\left(z_{4}-z_{1}\right)+y_{4}\left(z_{1}-z_{3}\right)\right]+ \\ x_{3}\left[y_{1}\left(z_{4}-z_{2}\right)+y_{2}\left(z_{1}-z_{4}\right)+y_{4}\left(z_{2}-z_{1}\right)\right]+ \\ x_{4}\left[y_{1}\left(z_{2}-z_{3}\right)+y_{2}\left(z_{3}-z_{1}\right)+y_{3}\left(z_{1}-z_{2}\right)\right] .\end{array}\right|$

Equation 12 is essentially a matrix which describes the behavior of $U_{d}(\mathbf{r})$ at each node in the element. In some sense it can be thought of as a calculation of the future distribution of the scattering given the source on each node.

We can calculate $\left\{w^{e}\right\}$ analytically by assuming that $U_{r i}$ varies linearly between the nodes of the element (a practical assumption since we use linear elements for the finite element weights):

$$
\begin{aligned}
w_{i}^{e}= & \int_{\Omega} \phi_{i}^{e}(\mathbf{r}) S(\mathbf{r}) d \Omega \\
= & \nu V^{e}\left(\frac{U_{r i_{i}}}{10}+\frac{U_{r i_{j}}}{20}+\frac{U_{r i_{k}}}{20}+\frac{U_{r i_{l}}}{20}\right) \\
& \quad-\quad-\frac{\beta}{4 \pi} \sum_{f=1}^{4}\left(\Delta_{f} \phi_{i}^{e}\left(\mathbf{m}_{f}\right)\left[\hat{\mathbf{n}}_{f}^{+} \cdot \mathbf{Q}_{1}^{e}\left(\mathbf{m}_{f}\right)\right]\right)
\end{aligned}
$$

where $f$ is a face on element $e$ and $\Delta_{f}, \mathbf{m}_{f}$ and $\hat{\mathbf{n}}_{f}^{+}$is the area, midpoint and normal for face $f$.

Finally, if any face $f$ of $e$ lies on the the boundary of the domain $\Gamma_{b}$ then the following terms are added to $\left[K^{e}\right]$ and $\left\{w^{e}\right\}$ which are derived from substituting the boundary condition (Equation 8) into the $\partial / \partial n^{+}$term on Equation 9.

$$
\begin{aligned}
K_{i j}^{s} & =\sum_{f=1}^{4}\left\{\begin{array}{cc}
\text { if } f \in \Gamma_{b} & \beta \mu_{t r} \frac{\Delta_{f}}{8}\left(1+\delta_{i j}\right) \\
\text { else } & 0
\end{array}\right. \\
w_{i}^{s} & =\sum_{f=1}^{4}\left\{\begin{array}{cc}
\text { if } f \in \Gamma_{b} & -\beta \mu_{t r} \frac{\Delta_{f}}{4 \pi} \phi_{i}^{e}\left(\mathbf{m}_{f}\right) \hat{\mathbf{n}}_{\mathbf{f}}^{-} \cdot \mathbf{Q}_{1}\left(\mathbf{m}_{f}\right) \\
\text { else } & 0
\end{array}\right.
\end{aligned}
$$

To generate the matrix for the entire FEM mesh, the matrices from all the elements are summed together. To map the local elemental matrices to the global one we apply an $N \times 4$ matrix, $\left[V^{e}\right]$, to $\left[K^{e}\right]$ in the form

$$
[K]=\sum_{e=1}^{M}\left[V^{e}\right] \cdot\left[K^{e}\right] \cdot\left[V^{e}\right]^{T}
$$

where $M$ is the number of elements and the elements of $\left[V^{e}\right]$ are zero except for elements $V_{i 1}^{e}, V_{j 2}^{e}, V_{k 3}^{e}$ and $V_{l 4}^{e}$, where $i$, $j, k$ and $l$ are the global node numbers of local nodes 1,2 , 3 and 4 . Similarly the global vector $\{w\}$ is built off the local element source vector as

$$
\{w\}=\sum_{e=1}^{M}\left[V^{e}\right] \cdot\left\{w^{e}\right\}
$$

\section{Biological and Geometrical Phantom}

The data shown in Figure 3a was derived from hand segmented MRI slices of the primary author's right forearm. For reference, a slice from this MRI dataset is shown in Figure 5. Unfortunately, MRI systems are not configured to image human arms as high resolution; as an alternative the dataset used in this paper was derived by inserting the arm into a knee coil on a 3 Tesla MRI system. This ad hoc configuration resulted in a rather noisy dataset which yielded a usable part approximately 1.5 inches in length. Although the MRI phantom presented here is smaller than we would prefer, it can still be used to accurately simulate NIR scatter. Furthermore the MRI was unable to capture the superficial veins seen in Figure $3 \mathrm{~b}$ due in part to the restricted blood flow during the data capture and the overall size of the structures. However, we were able to recreate these structures by modeling the visible surface veins on the original specimen.

We have also created a second dataset by hand, using anatomical data, in the finite element meshing software ANSYS. Our model (shown in Figure 6 includes both the ulnar and radial artery, superficial veins, the radius and ulna bones, and an outer "muscle" layer. We use this model later to demonstrate the effect of light placement and age effects on the NIR light propagation.

Although it is difficult to measure in vivo scattering values for internal human tissues many studies exist which measure the absorption coefficient $\mu_{a}$ and the reduced scattering coefficient $\mu_{s}^{\prime}$ in a variety of tissues at different wavelengths [6], [10], [15], [31]. The scattering properties we chose are shown in Table II were for wavelength $950 \mathrm{~nm}$. 


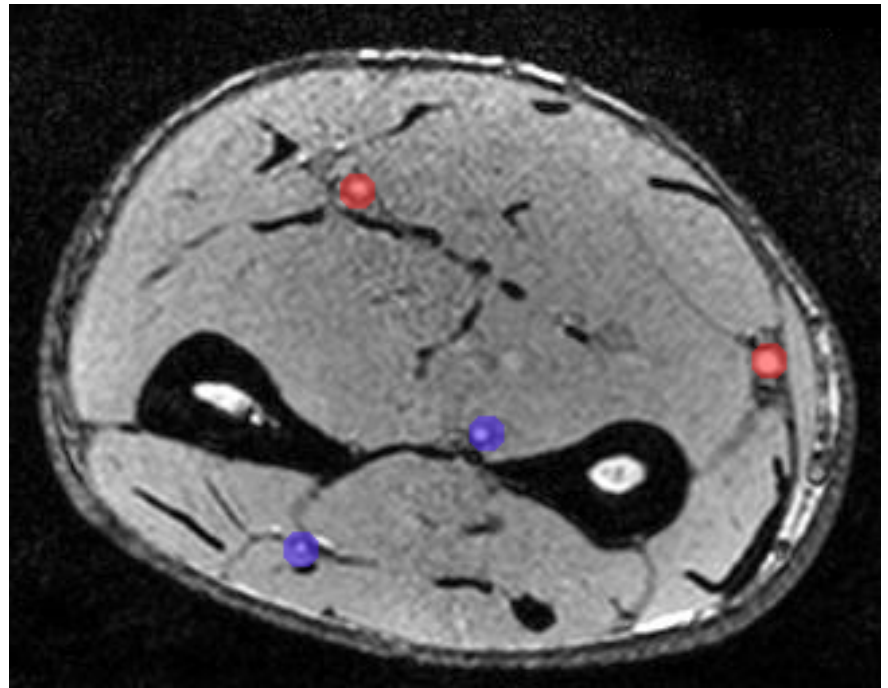

Fig. 5. A sample slice of a MRI of the author's forearm used in the biological phantom in Figure 3a. The two main arteries (ulnar and radial) are marked in red and the two veins are marked in blue.

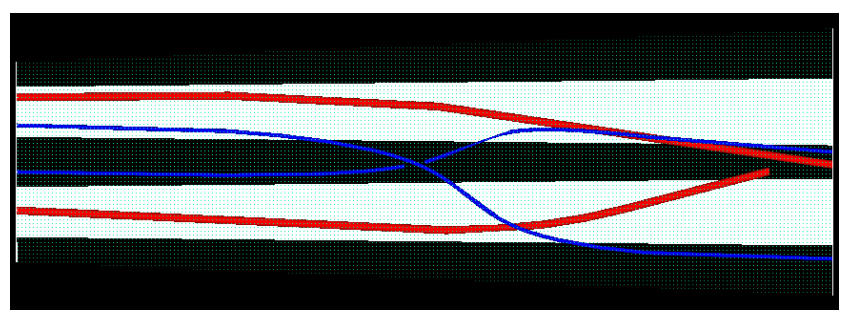

Fig. 6. ANSYS model for human forearm. Red vessels are arteries and blue vessels are superficial veins. Inner cylinders represent the radius and ulna bones.

\section{A. Optical Properties of Skin}

The performance of the IRIS vascular viewer is known to be affected by the age of the patient. In this section, we use the simulation tool to investigate the cause. NIR light is absorbed in the dermis mainly by water and collagen [42]. Although skin thickness decreases with age [40] the water content of the skin is greater with increasing age when expressed per unit weight [30]. Furthermore the proportion of insoluble collagen increases with age while that of soluble collagen decreases [18].

Since the total skin thickness in young men is $1.0-1.2 \mathrm{~mm}$ and young women $0.8-1.0 \mathrm{~mm}$ and decreases to $0.7-0.9 \mathrm{~mm}$ in both by age 70 [40] we model this absorption in the skin layer as part of the $U_{r i}$ source distribution.

Since the outer skin layer is relatively thin compared to

\begin{tabular}{c|c|c|c} 
Tissue & $\mu_{s}\left(\mathrm{~cm}^{-1}\right)$ & $\mu_{a}\left(\mathrm{~cm}^{-1}\right)$ & $g$ \\
\hline Bone [14] & 240 & 0.5 & 0.945 \\
Muscle [37] & $55.5^{\dagger}$ & 0.456 & 0.9 \\
Blood [10] & 2.84 & 505 & 0.992
\end{tabular}

TABLE II

OPTICAL PROPERTIES FOR THE SIMULATION SHOWN IN FIGURES 3 AND 8.

THE SOURCE WAVELENGTH IS ASSUMED TO BE 950NM. ( ${ }^{\dagger}$ ASSUMING

$$
g=0.9 . ;{ }^{\star} \text { WAVELENGTH }=960 \mathrm{NM} \text {.) }
$$

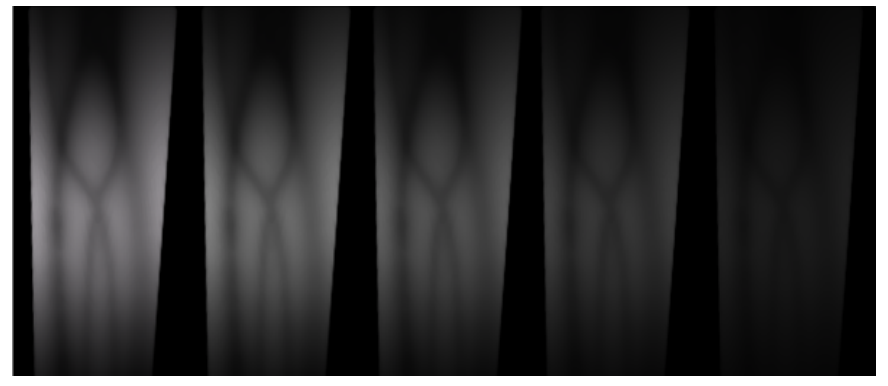

Fig. 7. Renderings of our arm dataset as the skin thickness is increased. The left image allows $100 \%$ of the NIR light into and out of the model while the right image reduces the NIR penetration to only $20 \%$.

the rest of the subsurface, its contribution is only affected as light enters and leaves the medium. Since our boundary condition assumes no inward directed diffuse intensity, the absorbing boundary layer will not have an effect on the diffuse intensity computations. This occurs since intensity cannot be reflected from the boundary, absorbed, then reflected back into the medium. Thus we model the effect of NIR absorption in the skin layer by scaling the reduced incident intensity by a value proportional to the rate of absorption. This is essentially a modification of the particular solution to Equation 2 such that we scale the initial intensity by some percentage:

$$
I_{r i}(\mathbf{r}, \hat{\mathbf{s}})=\Lambda(t) I_{r i}\left(\mathbf{r}_{0}, \hat{\mathbf{s}}\right) e^{-\int \mu_{t}(\mathbf{s}) \mathbf{s} d \mathbf{s}}
$$

Where $\mathbf{r}_{0}$ is the location of the light source and $\Lambda(t)$ is a parameter based percentage between $[0,1]$ which accounts for the NIR absorption in the skin. This term can be calculated by exponentially decaying the absorption coefficient for skin [19] across the depth of the skin

$$
\Lambda(t)=e^{-\delta(t) \mu_{a}(t)}
$$

where $\delta(t)$ is the thickness of the skin and $\mu_{a}(t)$ is the absorption cross section for that section of skin.

Likewise, in the final projection of $U_{d}(\mathbf{r})$ and $U_{r i}(\mathbf{r})$ to the detector we also scale the output intensity by this same parameter to account for the absorption of light exiting the skin.

\section{IMPLEMENTATION}

In this section we discuss the details of our implementation, namely the algorithm to create the $[K]$ matrix described in Equations 12 and 16, creation of the source vector $\{w\}$ given Equations 15 and 17, and a method for solving the system and displaying the results. Since the mesh is dense and system of equations so large we must be careful in the implementation to avoid any unnecessary calculations.

\section{A. Matrix $[K]$}

When constructing the matrix for the finite element system we are building the sparse global matrix $[K]$. However its construction is based on contributions from every element in the mesh. In general we loop through every element in the mesh, build a local $\left[K^{e}\right]$ matrix based on Equation 12 and 
boundary conditions in Equation 16, then add $\left[K^{e}\right]$ to the global matrix $[K]$ by mapping the local element numbers to the global ones as shown in Equation 18. This algorithm is shown in Algorithm 1.

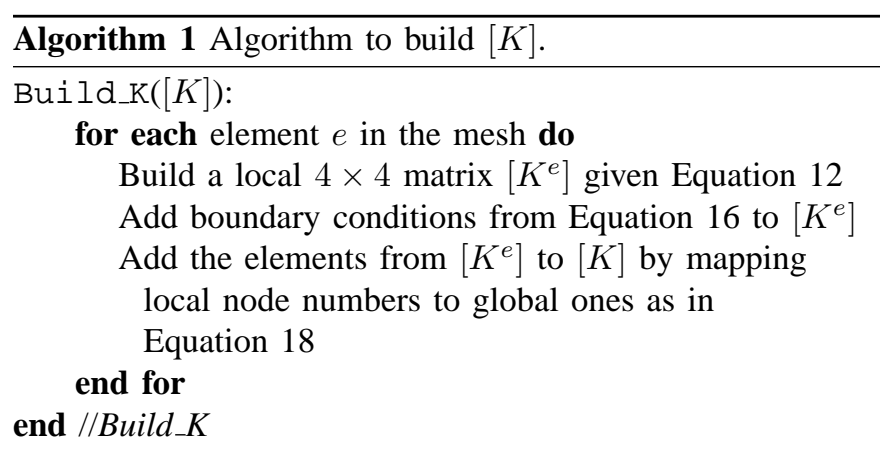

\section{B. Source Distribution $\{w\}$}

The source distribution is calculated from solving for $U_{r i}(\mathbf{r})$ at every node in the mesh (see Equation 3) then integrating across the elements as shown in Equation 15. Calculating $U_{r i}$ involves tracing a ray from the node to the light source and attenuating the source light based on the extinction cross section $\left(\mu_{t}\right)$ of the elements the ray passes through. Since it is possible to have different material properties in each element of the mesh we must calculate an intersection for each element the ray passes through, as opposed to the straight line distance from the node to the edge of the mesh. To avoid expensive intersection tests with all the elements in the node, we partition the domain containing all elements of the FE grid in an octree. The intersection task is then simplified by testing only those elements that reside in octants that intersect the ray. For a well balanced octree this reduces the number of potential intersection checks from linear to log complexity. The algorithm for building vector $\{w\}$ is shown in Algorithm 2.

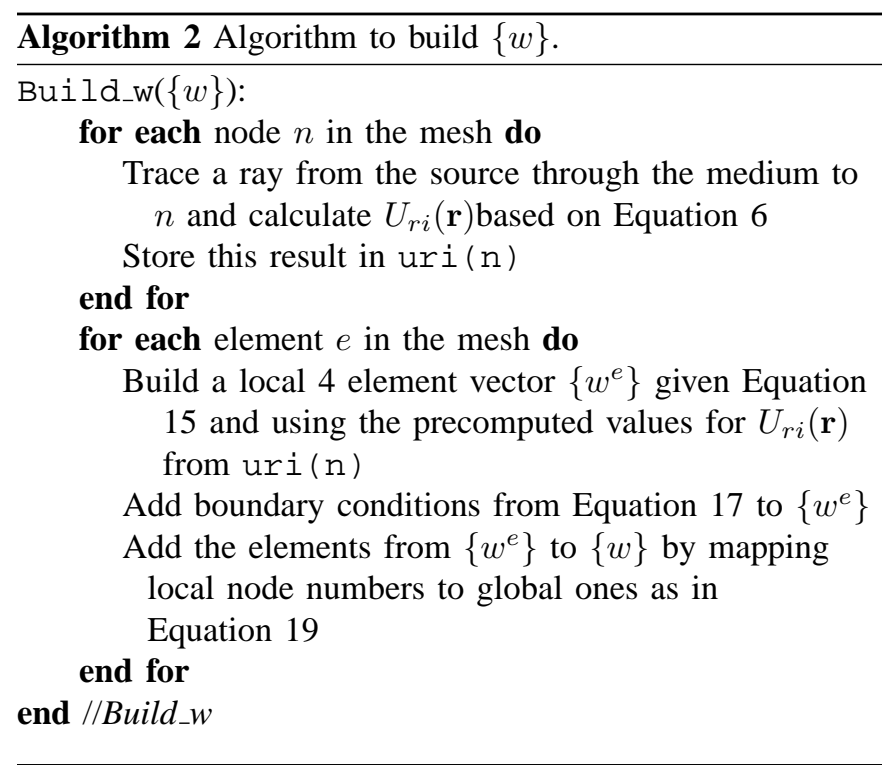

\section{Matrix Factorization of $[K]$}

Once we build the elemental global matrix and source vector we solve a linear system of the form $[K]\left\{U_{d}\right\}=$ $\{w\}$ to calculate the diffuse light distribution. Fortunately the elemental matrix of the diffusion equation is positive-definite, thus we can use Cholesky decomposition [33] to solve the system which is an economical method for decomposing $[K]$ into the form

$$
[K]=[L][L]^{T} .
$$

To solve this system one then solves the equation $[L]\{y\}=$ $\{w\}$, then $[L]^{T}\left\{U_{d}\right\}=\{y\}$. These solutions are trivial since both vectors can be directly solved using forward and backsubstitution.

To reduce non-zero fill in the factor $L$ we reorder the factorization with the METIS multilevel graph partitioning algorithm [25] in conjunction with sparse matrix library TAUCS [41]. Note that once the matrix is factored, one can change the location of the source and quickly recalculate the $U_{d}(\mathbf{r})$ diffusion distribution.

As long as the material properties in the mesh remain constant we can save the factor $[L]$ as a precomputation of the light scattering in the mesh to update the solution as the light source moves. Table III shows the timings for each step in the algorithm. If we reuse the factor $[L]$ this causes the time in column "Factor $+[K]$ Creation Time" to be a precalculation cost and successive solutions on the mesh would simply be the "Source Creation Time" plus "Solution Time."

\section{Rendering}

To render the simulated NIR images in this paper, we first solve for $U_{d}(\mathbf{r})$ at all points in the mesh then interpolate the solutions into a complete volume. If the nodal solutions are projected directly to the surface of the model using a texture mapped visualization approach, the solution tends to look rough and pixelated due to the tetrahedral element boundaries aliasing the solution. Instead we load our solved volume as a 3D volume of scalar intensities into Kitware's VolView volume visualization software. Allowing a bit of translucency in the volume generates more realistic renderings. Volume rendering the solution is essentially similar to modeling the subtle forward scattering effects as light diffuses out of the material which are not completely modeled by the diffusion equation.

\section{RESULTS AND DISCUSSION}

One advantage of our model is the ability to perform simulations which could not otherwise be done with real world subjects without difficulty. As an example, one experiment we performed was to transilluminate an absorption discontinuity which could be moved below the surface of an otherwise diffusely scattering object. As the discontinuity is moved the mean intensity increases due to more light being allowed to reach that area on the surface. Simultaneously, the standard deviation of the intensity histogram decreases, reflecting the fact that the intensity is distributed more evenly across the 


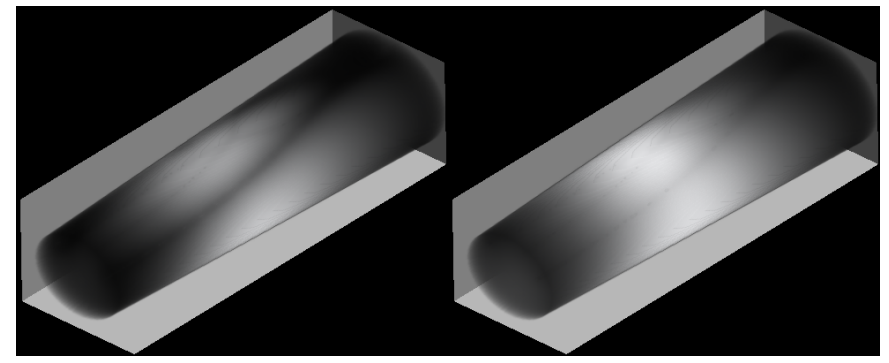

Fig. 8. This figure shows the solution of $U_{d}(\mathbf{r})$ on our finite element model shown in Figure 6. The arm on the left is lit from the bottom while the image on the right is lit from the top. The planes in the background are shown for perspective

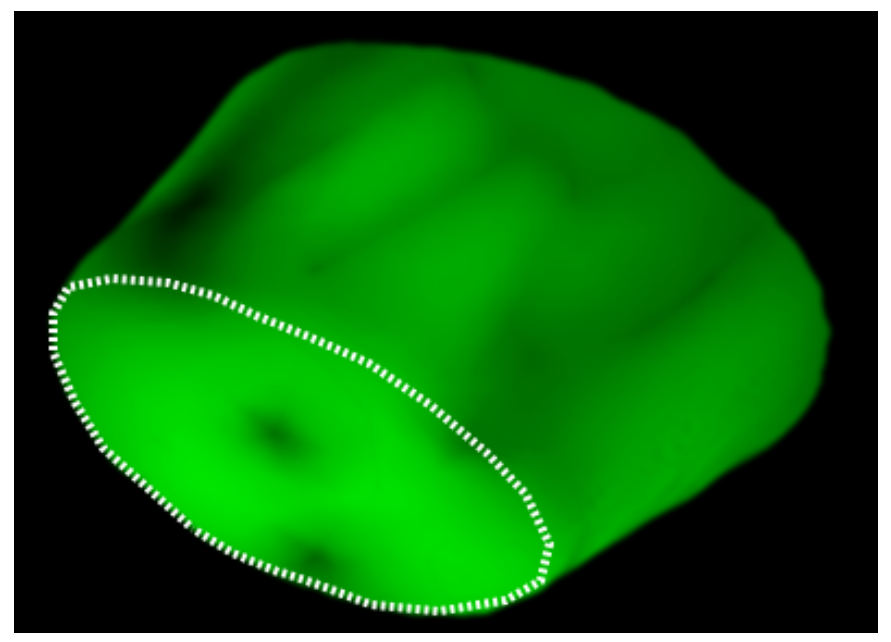

Fig. 9. This figure shows a perspective view of the MRI data at the edge of the dataset. Note that both the arteries and the veins are visible, however the shadowing caused by the veins is too deep beneath the surface to see through to the detector (top) side. The dotted circle is drawn around the end of the dataset for perspective.

surface. The images in Figures 10a and 10b demonstrate the visual aspects of this experiment while the image in Figure 10c shows our visibility metric as the discontinuity moves beneath the surface.

Figure 8 illustrates the effect of source placement on the rendering of the synthetic arm with both a top and bottom lit configuration. Finally, Figure 9 demonstrates the ability to simulate not only the visible surface light, but the complete intensity distribution in a volume with a perspective cutaway view of the MRI phantom.

It is important to note that the images rendered in this paper could be rendered using Monte Carlo photon tracing methods [21]. However, since diffusion dominates the photon propagation, many photons must be traced to accurately capture the diffusion effect. Thus, the model presented in this paper can be considered a computationally efficient technique for solving light distributions in media with high scatter and low absorption. Namely, the finite element solution to the diffusion approximation.

Some sample run times and matrix fill statistics are given in Table III. As mentioned previously, the "Factor $+[K]$ Creation Time" column can be thought of as a precomputation step if multiple simulations are run with a moving light source.
Saving the factorization reduces successive calculations by approximately half.

\section{CONCLUSIONS}

In this work we have presented a practical framework for solving light diffusion problems in three dimensions for the purpose of simulating light scattering in inhomogeneous materials. Many scattering models used in computer graphics literature are unable to capture the diffuse spread of light in inhomogeneous materials due to the high rate of scattering in the medium. We have shown that a finite element solution to the diffusion equation is able to not only accurately capture many subsurface scattering effects seen in real world NIR transillumination but provide the entire volumetric intensity distribution which would otherwise be unachievable with real world subjects. As part of our continuing work, we wish to not only use this model to help design NIR medical devices, but also refine the numerical phantoms used in this paper.

\section{ACKNOWLEDGMENTS}

We would like to thank to Dr. Steffen Sammet and Regina Koch at the Radiology Department at the Ohio State University for donating their time and equipment to capture the MRI images seen in this paper.

\section{REFERENCES}

[1] S. Arridge, M. Schweiger, M. Hiraoka, and D. Delpy. A finite element approach for modeling photon transport in tissue. Med. Phys., 20(2):299-309, Mar/Apr 1993.

[2] S. R. Arridge and J. C. Hebden. Optical imaging in medicine ii. modeling and reconstruction. Med. Phys., 42(841), 1997.

[3] E. Aydin, C. de Oliveira, and A. Goddard. A comparison between transport and diffusion calculations using a finite element-spherical harmonics radiation transport method. Med. Phys., 29(9), September 2002.

[4] G. Bal. Particle transport through scattering regions with clear layers and inclusions. Journal of Computational Physics, 180:659-685, 2002.

[5] D. A. Boas, M. A. Franceschini, A. K. Dunn, and G. Strangman. In Vivo Optical Imaging of Brain Function, chapter Non-Invasive imaging of cerebral activation with diffuse optical tomography, pages 194-216. CRC Press, 2002.

[6] B. Brooksby, H. Dehghani, B. Pogue, and K. Paulsen. Near-infrared (nir) tomography breast image reconstruction with A Priori structural information from mri: Algorithm development for reconstructing heterogeneities. IEEE Journal of Selected Topics in Quantum Electronics, 9(2):199-209, March/April 2003.

[7] P. Brown. An I.V. specialty team can mean savings for hospital and patient. Journal of the National Intravenous Therapy Association, 7(5):387-388, 1984

[8] N. Carr, J. Hall, and J. Hart. Gpu algorithms for radiosity and subsurface scattering. In $H W W S$ '03: Proceedings of the ACM SIGGRAPH/EUROGRAPHICS conference on Graphics hardware, pages 51-59, Aire-la-Ville, Switzerland, Switzerland, 2003. Eurographics Association.

[9] S. Chandrasekhar. Radiative Transfer. Dover Publications, Inc., 1960.

[10] W.-F. Cheong, S. Prahl, and A. Welch. A review of the optical properties of biological tissues. IEEE Journal of Quantum Electronics, 26(12):2166-2185, December 1990

[11] A. Cong and G. Wang. Multispectral bioluminescence tomography: Methodology and simulation. International Journal of Biomedical Imaging, 2006:1-7, 2006.

[12] C. Donner and H. W. Jensen. Light diffusion in multi-layered translucent materials. In ACM Transactions on Graphics (SIGGRAPH'2005), pages 1032-1039, Los Angeles, August 2005. ACM.

[13] B. Finlayson. The method of weighted residuals and variational principles, with application in fluid mechanics, heat and mass transfer. Academic Press, New York, 1972. 


\begin{tabular}{c|c|c|c|c|c}
$\begin{array}{c}\text { \# of } \\
\text { Elements }\end{array}$ & $\begin{array}{c}\text { Non-zeros } \\
\text { in }[K]\end{array}$ & $\begin{array}{c}\text { Non-zeros } \\
\text { in }[L]\end{array}$ & $\begin{array}{c}\text { Source } \\
\text { Creation } \\
\text { Time }\end{array}$ & $\begin{array}{c}\text { Factor }+ \\
{[K]} \\
\text { Creation } \\
\text { Time }\end{array}$ & $\begin{array}{c}\text { Solution } \\
\text { Time }\end{array}$ \\
\hline 5000 & 8261 & $4.30 \mathrm{e} 4$ & $1.187 \mathrm{~s}$ & $2.141 \mathrm{~s}$ & $0.046 \mathrm{~s}$ \\
\hline 16875 & 26416 & $3.20 \mathrm{e} 5$ & $5.156 \mathrm{~s}$ & $7.266 \mathrm{~s}$ & $0.172 \mathrm{~s}$ \\
\hline 40000 & 60921 & $8.09 \mathrm{e} 5$ & $17.172 \mathrm{~s}$ & $17.22 \mathrm{~s}$ & $0.562 \mathrm{~s}$ \\
\hline 78125 & 117026 & $2.65 \mathrm{e} 6$ & $37.937 \mathrm{~s}$ & $34.204 \mathrm{~s}$ & $1.563 \mathrm{~s}$
\end{tabular}

TABLE III

PROGRESSION FOR TIMINGS AND NON-ZERO FILL RATES AS MESH SIZE IS INCREASED. THESE RESULTS WERE GENERATED FROM A 1 CM ${ }^{2}$ CUBE WHICH WAS EVENLY MESHED. THE NON-ZEROS IN $[K]$ REFER TO THE NUMBER OF NON ZEROS GENERATED BY EQUATION 12 . NON-ZEROS IN $L$ REFER TO THE NUMBER OF ZEROS GENERATED IN THE CHOLESKY FACTORIZATION GIVEN IN EQUATION 22 AND GIVES INSIGHT TO THE MEMORY USAGE BASED ON THE ORIGINAL NUMBER OF UNKNOWNS. THE FACTOR TIME IS THE MEASURED TIME TO GENERATE $[K]=[L][L]^{T}$. THE SOLUTION TIME IS THE AMOUNT OF TIME TO CALCULATE $\left\{U_{d}\right\}$ FROM $[K]\left\{U_{d}\right\}=\{w\}$ GIVEN THE FACTORIZATION $[L]$.

[14] M. Firbank, M. Hiraoka, M. Essenpreis, and D.T. Delpy. Measurement of the optical properties of the skull in the wavelength range $650-950 \mathrm{~nm}$. Physics in Medicine and Biology, 38(4):503-510, April 1993.

[15] J. Fishkin, O. Coquoz, E. Anderson, M. Brenner, and B. Tromberg. Frequency-domain photon migration measurements of normal and malignant tissue optical properties in a human subject. Applied Optics, 36(1):10-20, January 1997.

[16] M.A. Franceschini and D. A. Boas. Noninvasive measurement of neuronal activity with near-infrared optical imaging. Neuroimage, 21(1):372-386, 2004.

[17] G. Gratton, P.M. Corballis, E. Cho, M. Fabiani, and D.C. Hood. Shades of gray matter: noninvasive optical images of human brain responses during visual stimulation. Psychophysiology, 32(5):505-509, September 1995.

[18] D. A. Hall. The Ageing of Connective Tissue. Academic Press, London, 1976.

[19] A. Hidenobu, E. Mariko, and Y Yukio. Depth profile of diffuse reflectance near-infrared spectroscopy for measurement of water content in skin. Skin Research and Technology, 11(1):27-35, 2005.

[20] A. Ishimaru. Wave Propagation and Scattering in Random Media. Academic Press, New York, 1978

[21] H. W. Jensen. Realistic Image Synthesis using Photon Mapping. AK Peters, 2001.

[22] H. W. Jensen and J. Buhler. A rapid hierarchical rendering technique for translucent materials. SIGGRAPH, 21(3):576-581, July 2005.

[23] H. W. Jensen, S. R. Marschner, M. Levoy, and P. Hanrahan. A practical model for subsurface light transport. In Proceedings of the 28th annual conference on computer graphics and interactive techniques, pages 511518. ACM Press, 2001

[24] K. Kalantar-Zadeh, E. Dunne, K. Nixon, K. Kahn, G.H. Lee, M. Kleiner, and F.C. Luft. Near infra-red interactance for nutritional assessment of dialysis patients. Nephrol Dial Transplant, 14(1):169-175, January 1999.

[25] G. Karypis and V. Kumar. A fast and high quality multilevel scheme for partitioning irregular graphs. SIAM J. Sci. Comput., 20(1):359-392, 1998.

[26] J. Kniss, S. Premoze, C. Hansen, and D. Ebert. Interactive translucent volume rendering and procedural modeling. In Proceedings of the conference on Visualization '02, pages 109-116. IEEE Computer Society, 2002.

[27] D. Laney, S. Callahan, N. Max, C. Silva, S. Langer, and R. Frank. Hardware-acceleated simulated radiography. In Visualization, pages 343-350. IEEE, 2005.

[28] J. Lee, S. Kim, and Y. Kim. Modeling of diffuse-diffuse photon coupling via a nonscattering region: a comparative study. Applied Optics, 43(18), June 2004.

[29] R. Liniger. Pediatric peripheral IV insertion success rates. Pediatric Nursing, 29(5):351-354, 2003.

[30] R. Marks and S.P. Barton. The significance of the size and shape of corneocytes. In R. Marks and G. Plewig, editors, Stratum Corneum. Springer Verlag, Berlin, 1983.

[31] G. Marquez, L. Wang, S. P. Lin, J. Schwartz, and S. Thomsen. Anisotropy in the absorption and scattering spectra of chicken breast tissue. Applied Optics, 37(4):798-804, 1998.
[32] N. Max. Optical models for direct volume rendering. IEEE Transactions on Visualization and Computer Graphics, 1(2):99-108, 1995.

[33] W. H. Press, S. A. Teukolsky, W. T. Vetterling, and B. P. Flannery. Numerical Recipes in C. Press Syndicate of the University of Cambridge, 1992.

[34] K. Riley, D. Ebert, C. Hansen, and J. Levit. Visually accurate multifield weather visualization. In Proceedings IEEE Visualization, pages 279-286, October 2003.

[35] K. Sakatani, S. Chen, W. Lichty, H. Zuo, and Y.P. Wang. Cerebral blood oxygenation changes induced by auditory stimulation in newborn infants measured by near infrared spectroscopy. Early Human Development, 55(3):229-236, July 1999.

[36] M. Schweiger, S. Arridge, M. Hirako, and D. Delpy. The finite element method for the propagation of light in scattering media: Boundary and source conditions. Med. Phys., 22(11):1779-1792, November 1995.

[37] C.R. Simpson, M. Kohl, M. Essenpreis, and M. Cope. Near infrared optical properties of ex-vivo human skin and subcutaneous tissues measured using the monte carlo inversion technique. Physics in Medicine and Biology, 43:2465-2478, 1998.

[38] P.-P. Sloan, J. Kautz, and J. Snyder. Precomputed radiance transfer for real-time rendering in dynamic, low-frequency lighting environments. In SIGGRAPH, July 2002.

[39] G. Strang and G. J. Fix. An Analysis of the Finite Element Method. Wellesley-Cambridge Press, 1988

[40] C.Y. Tan, B. Statham, R. Marks, and P.A. Payne. Skin thickness measurement by pulsed ultrasound: its reproducibility, validation and variability. The British Journal of Dermatology, 106(6):657-667, June 1982.

[41] S. Toledo, D. Chen, and V. Rotkin. Taucs: A library of sparse linear solvers. http://www.tau.ac.il/ stoledo/taucs/.

[42] J.A. Viator, B. Choi, G.M. Peavy, S. Kimel, and J.S Nelson. Spectra from 2.5-15 microm of tissue phantom materials, optical clearing agents and ex vivo human skin: implications for depth profiling of human skin. Phys. Med. Biol., 48(2):N15-24, January 2003.

[43] B. Wilson, E. Sevic, M. Patterson, and B. Chance. Time-dependent optical spectroscopy and imagin for biomedical applications. Proceedings of the III, 80(6), 1992.

[44] X. Xu, W. Zhu, V. Padival, M. Xia, X. Cheng, R. Bush, L. Christenson, T. Chan, T. Doherty, and A. Iatridis. Validation of nirs in measuring tissue hemoglobin concentration and oxygen saturation using benchtop and isolated limb model. In Proceedings of SPIE, volume 4955, pages 369-378, 2003.

[45] O. C. Zienkiewicz and R. L. Taylor. The Finite Element Method. McGraw-Hill, 1988. 


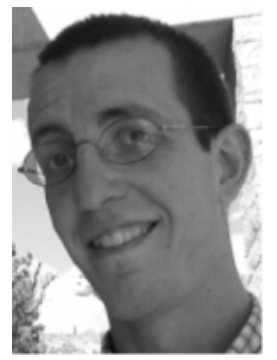

Richard Sharp Richard Sharp graduated from Ohio State University in 2006 with a doctorate in Computer Science. He is now a Senior Lecturer at Ohio State University and continues to pursue research interests including physics modeling, bioinformatics and software visualization.

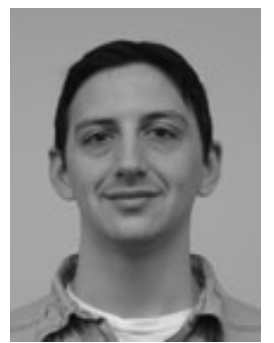

Jacob Adams Jacob Adams is a Ph.D. candidate at the department of Electrical and Computer Engineering at the Ohio State University. His primary research interests are in numerical methods and bioelectromagnetics.

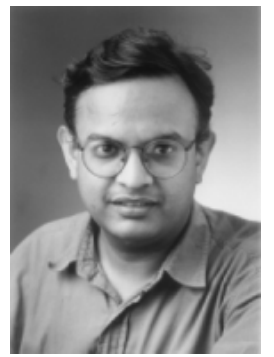

Raghu Machiraju Raghu Machiraju is an Associate Professor of Computer Science and Engineering at The Ohio State University since January 2000. Earlier, he was at the NSF Engineering Research Center, Mississippi State University. His research interests include rendering, feature detection and computational methods and modeling.

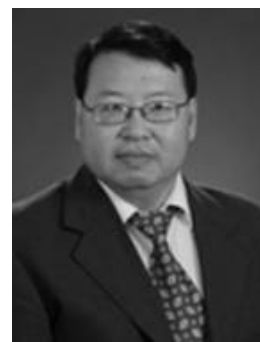

Robert Lee Dr. Lee's primary area of research interest is in numerical techniques for electromagnetics, specifically on differential-based techniques such as the finite element and finite difference methods. $\mathrm{He}$ has also worked on hybrid methods, boundary truncation techniques and error analysis of FEM. He has used his techniques to solve problems in jet inlet scattering, mine detection antennas and coils for high field MRI.

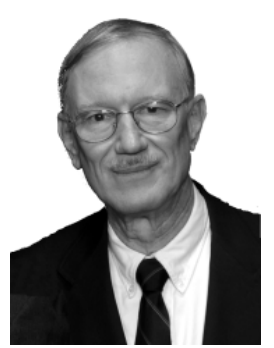

Robert Crane Robert Crane graduated from Ohio State University with a doctorate in Materials Science in 1969. He spent the next 33 years working for the Air Force Research Laboratory in a number of diverse technical disciplines - nondestructive inspection, solid mechanics, micro-electromechanicalsystems (MEMS), and near infrared optics for defense against laser weapons. He currently works for Infrared Imaging Systems Inc. as their chief scientist. (a)
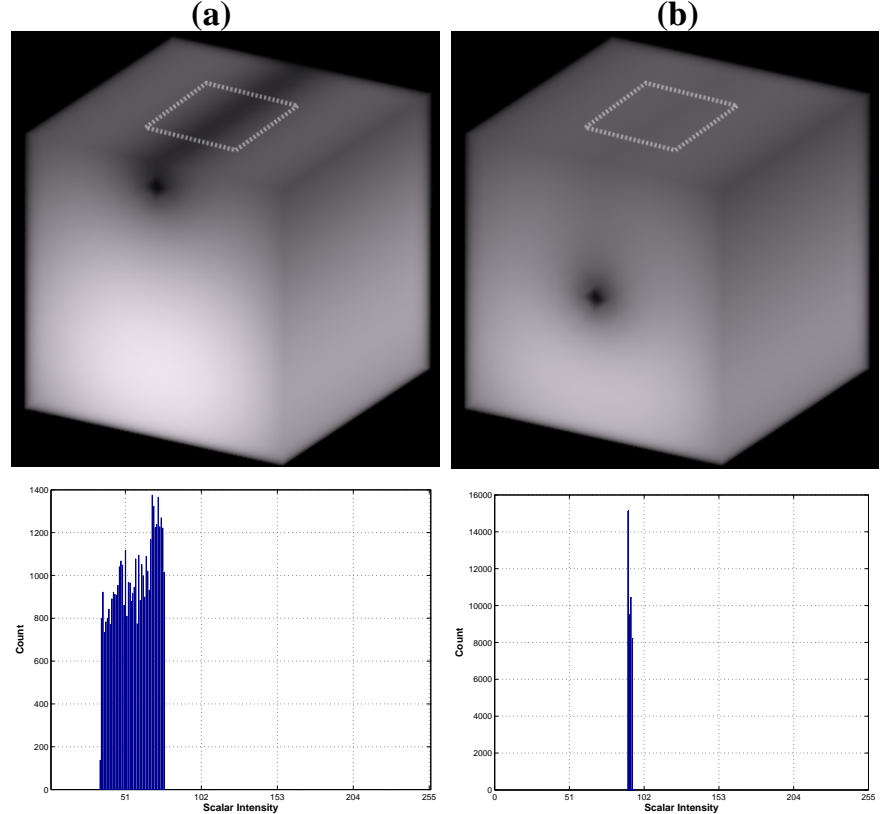

(c)

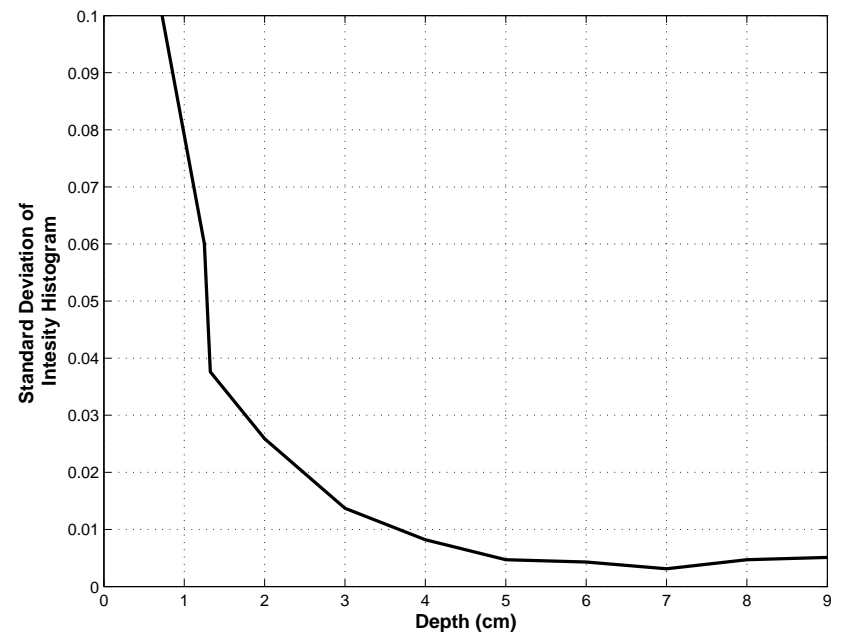

Fig. 10. This figure illustrates our metric for determining visibility of a subsurface structure in a $10 \mathrm{~cm}^{3}$ medium. Column (a) places the discontinuity at $1 \mathrm{~cm}$ while column (b) places it at $5 \mathrm{~cm}$. The histogram plots on the bottom row show the intensity of the pixels around the white boxes drawn on the top surface of the cubes. Notice as the discontinuity sinks the average intensity increases while the standard deviation of the histogram decreases. Figure (c) shows how the standard deviation of intensity varies as the discontinuity moves below the surface. $\mu_{s}=10 \mathrm{~cm}^{-1} \mu_{a}=0.1 \mathrm{~cm}^{-1}$. The discontinuity visibility drops significantly after $1.5 \mathrm{~cm}$. 